\title{
Design for Temperature Measurement System Based on Smart Phone and Infrared Thermal Camera Core
}

\author{
Leizi Jiao, Xiande Zhao, Daming Dong* \\ National Engineering Research Center for Information Technology in Agriculture \\ Beijing Academy of Agriculture and Forestry Sciences, BAAFS \\ Beijing, China \\ E-mail: dongdm@nercita.org.cn
}

Keywords: Thermal imaging camera core; Smart phone; Bluetooth; Serial port

\begin{abstract}
Commercial thermal infrared imager with large volume and high cost usually captured the infrared thermal image with automatic gain in order to enhance contrast of the image. The infrared thermal image captured with automatic gain could not represent the original image information corresponding to the temperature of object surface. So this treatment limited its application in the measurement of absolute temperature. This design used smart phone and infrared thermal camera core with small volume and low cost for developing temperature measurement system transmitted data through the blue tooth serial port pass through module. The small and convenient character of the system and ability of obtaining the original image information corresponding to the temperature of object surface enhanced the potential in practical application.
\end{abstract}

\section{Introduction}

Infrared thermal imaging is a testing technology which transforms thermal radiation of the object surface into the thermal image using infrared focal plane array. The noncontact and non-destructive character of infrared thermal image along with rapid online usability made it play a major role in temperature mapping of essential process and apply widely in the agriculture and food [1]. Such as plant stress [2-3], estimation of stomatal conductance for aiding to irrigation decision[4-5], defect detection and surface quality of fruit [6-13], detection of insect infestation in the process of grain stock [14-16], determination of foreign bodies in food[17-18]. Infrared thermal imaging cameras used in the above research work were usually commercial instruments, such as Thermal camera 384, Thermal camera SC500, Thermal camera AGEMA 570, thermal camera Model 760 and so on. Although these instruments have good performance, automatic gain was usually used in order to obtain excellent contrast of infrared thermal image, therefore, these images didn't represent the original image information corresponding to the surface temperature of object. In addition, these instruments had a high cost and needed to operate professionally. These disadvantages greatly limited its application in the absolute temperature measurement.

Thermal imaging camera core with low cost and small volume could conveniently access to the original infrared thermal image corresponding to the surface temperature of the object. These advantages can develop an inexpensive and small system for temperature measurement. Rapid development and increasingly fierce competitive environment have urged the cost of smart phone more and more cheap as well as the perfect performance.

According to the present situation, the paper aim to develop a low-cost thermal infrared temperature measurement system based on smart phone and infrared thermal camera core communicated with each other by the Bluetooth serial port pass through module. 


\section{Material and Method}

\section{A. Material}

Tau2 160 a $160 \times 128$ core manufactured by the FLIR was used in this design. This core was a long-wavelength uncooled microbolometer camera designed for application of needing absolute minimum size and power consumption. An asynchronous serial interface is provided by the core and baud rate of communication between the infrared thermal core and smart phone is set at the 57600 . Smart phone was used Mi 2S manufactured by the XiaoMi company. Bluetooth serial port pass through module used for the communication between the smart phone and the infrared thermal core was bought from the IRXON Electronics Co, .Ltd. This module uses higher-speed USB 2.0 standard and can transform serial port protocol into blue tooth protocol. The baud rate of the module for communication was also set at 57600. The schematic diagram of the temperature measuring system based on infrared thermal camera core and smart phone was shown in figure 1.

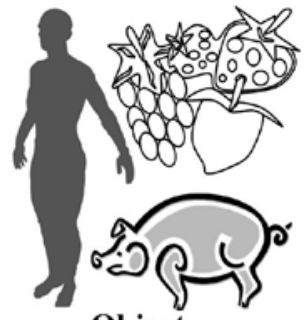

Object

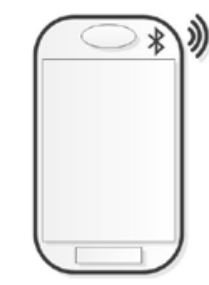

Smartphone

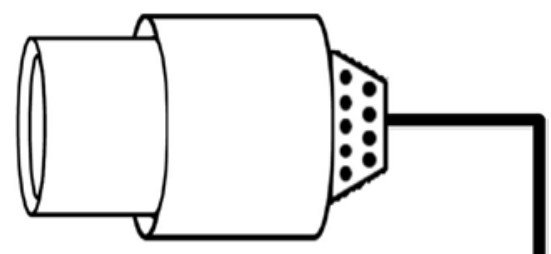

Thermal Imaging Camera Core

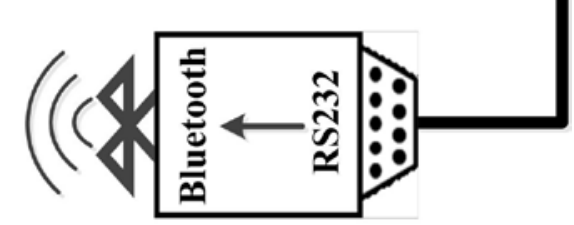

Bluetooth Serial Port Passthrough Module

Figure1. Schematic diagram of the temperature measuring system based on infrared thermal camera core and smart phone

\section{B. Method}

The pictures of the infrared thermal camera core, smart phone and blue tooth serial port pass through module were shown in figure 2. The Bluetooth of the smart phone and the power key of the blue tooth serial port pass through module were switched on, so that they could connect with each other depending on the same communication protocols. The original infrared thermal image extracted from the data received by the smart phone from the infrared thermal camera core was shown in the application software developed in the smart phone. 


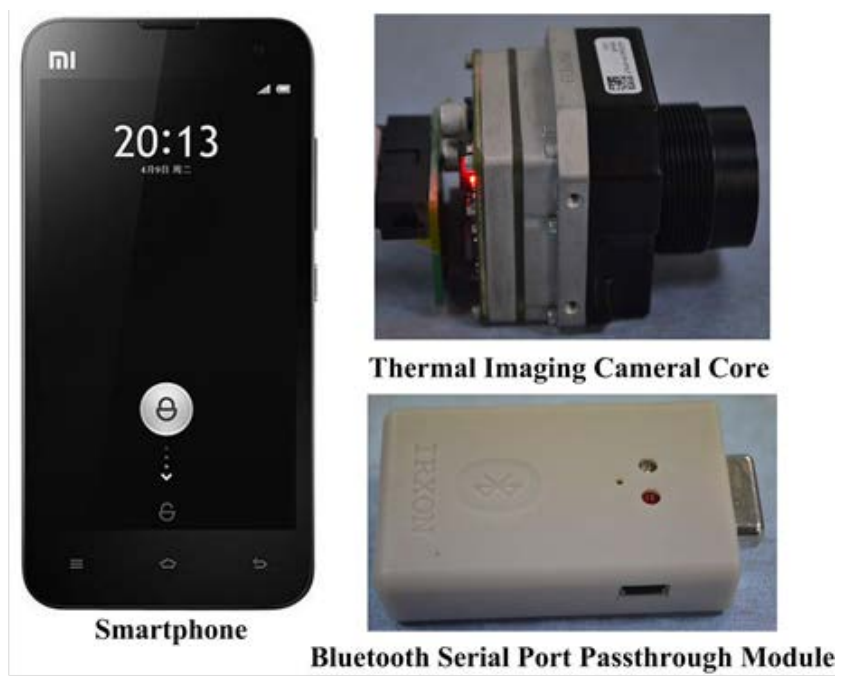

Figure 2. Product photos used in the temperature measuring system

\section{Conclusion}

The communication between smart phone and infrared thermal camera core was successful in the test experiment. The data delivered from the infrared thermal camera core could be received correctly by the application software developed in the smart phone. As shown in figure 3, the original infrared thermal image could be restored integrally from the received data by the application software from the infrared thermal camera core according to the format of the data. So the system feasibility was demonstrated through the monitoring experiment of human body. Relative to dedicated data acquisition terminal usually used in the instrument system, smart phone widely used in today's society was employed as a data acquisition terminal. Through the temperature measurement system developed in this design only carried out the preliminary design and validation, compared with the existing commercial thermal imager, the characters of small volume and low cost had a serious advantage in the practical application.

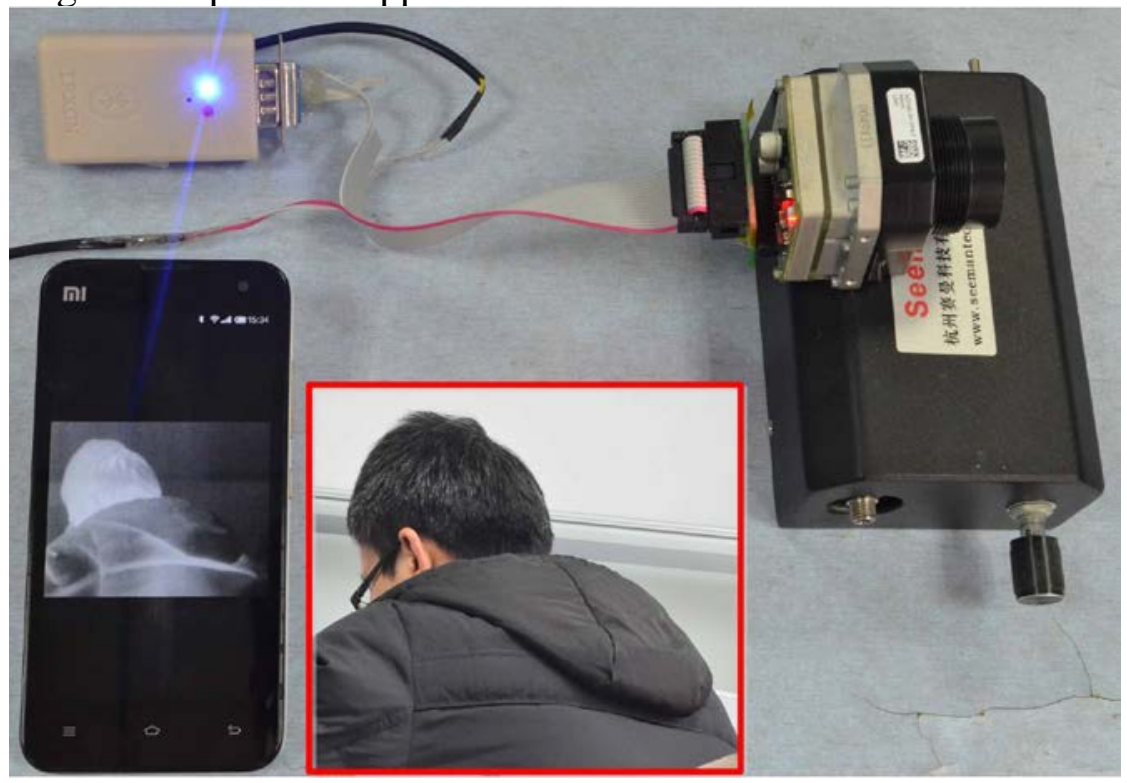

Figure 3. Experiment for monitoring human body using the developed infrared thermal temperature system

\section{Acknowledgment}

This work was supported by Beijing Natural Science Foundation (No. 4131002). 


\section{References}

[1] R. Vadivambal and D. S. Jayas, "Applications of Thermal Imaging in Agriculture and Food Industry-A Review," Food and Bioprocess Technology, vol. 4, pp. 186-199, 2011.

[2] I. Leinonen and H. G. Jones, "Combining thermal and visible imagery for estimating canopy temperature and identifying plant stress," Journal of Experimental Botany, vol. 55, pp. 1423-1431, 2004.

[3] M. Stoll and H. G. Jones, "Thermal imaging as a viable tool for monitoring plant stress," JOURNAL INTERNATIONAL DES SCIENCES DE LA VIGNE ET DU VIN, vol. 41, pp. 77-84, 2007.

[4] H. G. Jones, "Use of infrared thermometry for estimation of stomatal conductance as a possible aid to irrigation scheduling," Agricultural and Forest Meteorology, vol. 95, pp. 139 - 149, 1999.

[5] H. G. Jones, "Use of thermography for quantitative studies of spatial and temporal variation of stomatal conductance over leaf surfaces," Plant, Cell \& Environment, vol. 22, pp. 1043 - 1055, 1999.

[6] M. P. Fuller and W. Wisniewski, "The use of infrared thermal imaging in the study of ice nucleation and freezing of plants". vol. 23, pp. 81 - 89, 1998.

[7] P. Baranowski and W. Mazurek, "Detection of physiological disorders and mechanical defects in apples using thermography," INTERNATIONAL AGROPHYSICS, vol. 23, pp. 9-17, 2009.

[8] P. Baranowski, W. Mazurek, B. Witkowska-Walczak, and C. Sławiński, "Detection of early apple bruises using pulsed-phase thermography," Postharvest Biology and Technology, vol. 53, pp. 91-100, 2009.

[9] P. Baranowski, J. Lipecki, W. Mazurek, and R. T. Walczak, "Detection of watercore in 'Gloster' apples using thermography," Postharvest Biology and Technology, vol. 47, pp. 358-366, 2008.

[10] D. M. Bulanon, T. F. Burks and V. Alchanatis, "Study on temporal variation in citrus canopy using thermal imaging for citrus fruit detection," Biosystems Engineering, vol. 101, pp. 161-171, 2008.

[11] E. A. Veraverbeke, P. Verboven, J. Lammertyn, P. Cronje, J. De Baerdemaeker, and B. M. Nicolaï, "Thermographic surface quality evaluation of apple," Journal of Food Engineering, vol. 77, pp. 162-168, 2006.

[12] D. Stajnko, M. Lakota and M. Hočevar, "Estimation of number and diameter of apple fruits in an orchard during the growing season by thermal imaging," Computers and Electronics in Agriculture, vol. 42, pp. 31-42, 2004.

[13] J. Varith, G. M. Hyde, A. L. Baritelle, J. K. Fellman, and T. Sattabongkot, "Non-contact bruise detection in apples by thermal imaging," Innovative Food Science \& Emerging Technologies, vol. 4, pp. 211-218, 2003.

[14] A. Manickavasagan, D. S. Jayas and N. D. G. White, "Thermal imaging to detect infestation by Cryptolestes ferrugineus inside wheat kernels," Journal of Stored Products Research, vol. 44, pp. 186-192, 2008.

[15] M. Emekci, S. Navarro, E. Donahaye, M. Rindner, and A. Azrieli, "Respiration of Tribolium castaneum (Herbst) at reduced oxygen concentrations," Journal of Stored Products Research, vol. 38, pp. 413 - 425, 2002.

[16] K. A. Damcevski, P. C. Annis and C. J. Waterford, "Effect of grain on apparent respiration of adult stored-product Coleoptera in anair-tight system: implications for fumigant testing,". vol. 34, 1998, pp. 331-339.

[17] P. Meinlschmidt and V. Maergner, "Detection of foreign substances in food using thermography". Proc. SPIE 4710, March 15, 2002.

[18] G. Ginesu, D. D. Giusto, V. Margner, and P. Meinlschmidt, "Detection of foreign bodies in food by thermal image processing," Industrial Electronics, IEEE Transactions on, vol. 51, pp. 480490, 2004. 